\title{
Trivium
}

Revue franco-allemande de sciences humaines et sociales - Deutsch-französische Zeitschrift für Geistesund Sozialwissenschaften

$13 \mid 2013$

Entre morale, politique et religion : la cohésion sociale selon Emile Durkheim

\section{Das »Durkheimsche Dilemma« in der Soziologie der Moral}

\section{Bruno Karsenti}

Traducteur : Michael Bischoff

\section{OpenEdition \\ Journals}

Édition électronique

URL : http://journals.openedition.org/trivium/4447

DOI : $10.4000 /$ trivium.4447

ISSN : 1963-1820

Éditeur

Les éditions de la Maison des sciences de l'Homme

\section{Référence électronique}

Bruno Karsenti, « Das »Durkheimsche Dilemma« in der Soziologie der Moral », Trivium [Online], 13

2013, online erschienen am 28 Februar 2013, abgerufen am 08 September 2020. URL : http:// journals.openedition.org/trivium/4447 ; DOI : https://doi.org/10.4000/trivium.4447

Ce document a été généré automatiquement le 8 septembre 2020.

Les contenus des la revue Trivium sont mis à disposition selon les termes de la Licence Creative Commons Attribution - Pas d'Utilisation Commerciale - Pas de Modification 4.0 International. 


\title{
Das »Durkheimsche Dilemma« in der Soziologie der Moral
}

\author{
Bruno Karsenti \\ Traduction : Michael Bischoff
}

\section{NOTE DE L'ÉDITEUR}

Wir danken Herrn Bruno Karsenti sowie dem Verlag Economica für die freundliche Genehmigung, diesen Artikel in deutscher Übersetzung zu publizieren.

Nous remercions M. Bruno Karsenti ainsi que la maison d'édition Economica de nous avoir accordé l'autorisation de traduire ce texte pour le présent numéro.

1 Wenn es ein Thema gibt, bei dem Durkheim immer wieder in die Nähe der Philosophie kommt, dann ist es die Moral. $\mathrm{Zu}$ moralischen Fragen bringt die Soziologie eine einzigartige, im eigentlichen Sinne wissenschaftliche Klärung, die sie von anderen, vor ihrer Entstehung vorgetragenen Begründungsversuchen unterscheidet. Gleichwohl geht sie nur insofern über die klassischen Nachforschungen der praktischen Philosophie hinaus, als sie ihrerseits philosophisch bestimmt, was man unter einer moralischen Tatsache zu verstehen hat - in Anlehnung an den umfassenderen Begriff der sozialen Tatsache und dessen Erklärungsebene. Für den Soziologen handelt es sich hier um einen strategischen Ort, an dem seine Wissenschaft sich zu beweisen hat. Dort findet die Emanzipation der Soziologie ihre Rechtfertigung, aber das gelingt ihr nur, indem sie die Frage der Moral in anderen als den bisher benutzten philosophischen Begriffen formuliert. In dieser Hinsicht war der Vortrag »Bestimmung der moralischen Tatsache«, den er 1906 vor der Société française de Philosophie hielt, ein äußerst gewagtes und letztlich entscheidendes Unterfangen. Durkheim wagte sich hier auf ein Gebiet, auf dem er, wie er sehr wohl wusste, nicht ganz zuhause war, um die Besonderheit seines Vorhabens sichtbar $\mathrm{zu}$ machen und dessen unvermeidliche Auswirkungen auf das Fachgebiet aufzuzeigen, aus dem es ursprünglich hervorgegangen war. Nach der Entstehung der Soziologie kann die Philosophie nicht unverändert bleiben. Und die Veränderung, die sie erfährt, zeigt sich zuerst und vor 
allem im praktischen Bereich, in dem, was man unter "moralischen Tatsachen« verstehen muss.

2 F.-A. Isambert hat gezeigt, welche subversive Kraft allein schon in dem Ausdruck steckt. Er erinnert an die Vorläufer - vor allem bei Wundt, den Durkheim schon früh gelesen hat, wie sein Aufsatz über »La morale positive en Allemagne ${ }^{1}$ beweist - und arbeitet dessen spezifisch Durkheimsche Bedeutung heraus: Es geht nicht darum, die Gesellschaft als "Ort der Variationen der Moral« zu begreifen, sondern den sozialen Charakter dieser Variationen zu erkennen, »die Bestimmungen zu berücksichtigen, die den moralischen Tatsachen aufgrund ihres sozialen Charakters zukommen ${ }^{2}{ }^{2}$ So kommt denn auch dem Ausdruck »Bestimmung« im Titel seines Vortrags von 1906 eine besondere Bedeutung zu. Eine Wissenschaft der moralischen Tatsachen statt der Moral im Sinne eines Wesens, für das die geschichtlich-sozialen Formen jeweils nur Illustrationen darstellen, ist deshalb möglich, weil die moralischen Tatsachen gerade auch in ihrer Vielfalt einer wissenschaftlichen Erklärung zugänglich sind. Diese Erklärung liefert die Soziologie und vermag nur die Soziologie zu liefern, weil diese Tatsachen sozial determiniert sind. Kurz gesagt, die soziale Bestimmung der moralischen Tatsachen geht nicht nur über die widersprüchlichen philosophischen Ansätze der Moraltheorien hinaus, sondern auch über die noch mit essentialistischen Voraussetzungen arbeitenden Entwürfe einer »Wissenschaft der Moral«, für die Wundts Ansatz nur ein Beispiel ist.

3 Hier zeichnen sich also die Grundlagen einer Soziologie der Moral ab, die eine heute sehr lebendige Reflexion speist, von der Patrick Pharos kürzlich erschienenes Buch Morale et sociologie ein exemplarisches Zeugnis ablegt. Diese Grundlagen sind allerdings unvollständig und zerbrechlich, wenn man Pharo und vor ihm Isambert glauben will. Denn Durkheims Sicht hatte von Anfang an mit einem Dilemma zu kämpfen, aus dem sie nicht herausfand und das letztlich mit ihren positivistischen Prämissen zusammenhing. Wegen des externen Ansatzes, dem sie den Vorzug gab und der ja gerade in der Idee der "Tatsache" seinen Ausdruck fand, verschrieb sie sich einer deskriptiven Erforschung der Sitten und der darin erkennbaren sozialen Zwänge, ohne diese Forschung auch mit dem im eigentlichen Sinne subjektiven Pol der Moral zu verbinden, den sie an anderer Stelle durchaus aufzuzeigen vermochte: dem des subjektiven Verhältnisses zu den Werten, das seinen Ausdruck weniger in der von außen zu beobachtenden moralischen Tatsache als in den moralischen Handlungen und Urteilen findet, die sich in deren interner Struktur widerspiegeln. Inwiefern ist diese interne Struktur auf ihrer eigenen Ebene zugänglich für ein soziologisches Herangehen? Und in welchem Verhältnis steht sie zu den geschaffenen, der Beschreibung zugänglichen Phänomenen? In dieser Hinsicht scheint Webers Soziologie - gerade weil sie sich auf soziales Handeln konzentriert - andere einschlägige Lösungen anzubieten, und so kann man verstehen, dass Parsons sich vor allem davon inspirieren ließ. Eine von Weber zu Parsons führende Linie zeichnet sich an deren Schnitt- oder Berührungspunkt mit der Durkheimschen Soziologie ab, da beide doch - mit Isambert zu sagen - den Versuch unternahmen, den "Gordischen Knoten zu durchschlagen « ${ }^{3}$ und zum Subjekt zurückzukehren, das seinem Wesen nach als sozialer Akteur verstanden wurde. Dadurch ließen sie eine für die Soziologie der Moral konstitutive Mehrdeutigkeit deutlicher hervortreten, von der keineswegs sicher ist, ob sie jemals aufgelöst werden kann, und die darin besteht, dass ihr Objekt als im eigentlichen Sinne soziales Objekt sich ständig in Richtung einer subjektiven Dimension verflüchtigt, in 
der die moralische Erfahrung sich konstituiert und die sich nicht durch die Hypothese einer Internalisierung äußerer Regeln erklären lässt, weil hier zu berücksichtigen ist, dass in diesem Bereich auch eine "praktische Überzeugung " ${ }^{4}$ zum Zuge kommt - wie Durkheim selbst erkannte, als er nach dem Element suchte, das er als ein der Tatsache innwohnendes "Erstrebenswertsein« beschrieb.

Hier wäre es sinnvoll, sich noch einmal anzusehen, was die Bannerträger der heutigen Moralsoziologie als das »Durkheimsche Dilemma $\aleph^{5}$ bezeichnen. Dazu ist es erforderlich, auf ein philosophisches Fundament zurückzukommen, wie es vor allem in zwei Texten von 1906 und 1911 erkennbar wird: "Bestimmung der moralischen Tatsache« und "Werturteile und Wirklichkeitsurteile.$^{6}{ }^{6}$ Dieses Vorgehen findet seine Rechtfertigung in Durkheims Verständnis des Ausdrucks "Bestimmung" (détermination) in seiner Anwendung auf die moralische Tatsache im Singular. Kein Zweifel, dass der Soziologe ganz bewusst mit den Worten gespielt hat. Dass die moralischen Tatsachen im Plural in ihrer variablen empirischen Realität sozial determiniert seien, das versucht die Soziologie als eine empirische Wissenschaft, die ein Phänomen beobachtet und in eine gewisse Kausalordnung einfügen möchte, ja gerade zu beweisen. Und sie beweist es einfach durch das Wissen, das sie hervorbringt. Die moralische Tatsache in ihrer Allgemeinheit als determiniert auszuweisen gehört dagegen in den Bereich einer anderen Zielsetzung, die man durchaus als philosophisch bezeichnen darf, sofern man das damit verbundene philosophische Tun nur präziser kennzeichnet. Einerseits wird man zugeben müssen, das Durkheim hier eine Rückwendung vollzieht oder zumindest einen Augenblick innehält, um seinen Ansatz zu verdeutlichen. Aber zugleich weist er darauf hin, dass man diese Verdeutlichung nicht als vorwissenschaftliches oder der eigentlichen Wissenschaft vorausliegendes Vorgehen verstehen darf, das auf einer fundamentaleren Ebene läge als die praktische Tätigkeit des Wissenschaftlers. Die moralische Tatsache bestimmen heißt nicht, sie zu konstituieren, und erst recht nicht, die Moral zu konstituieren. Vielmehr fragt man dabei, wie die Tatsache sich bestimmt, wie sie in den moralischen Handlungen und Urteilen der sozialen Subjekte bestimmt wird. Wir befinden uns also im Herzen des Dilemmas, das spätere soziologische Ansätze ausmachten. Die philosophische "Pause«, die Durkheim sich erlaubte, ist nicht unabhängig von den Fragen, die er sich als Soziologe stellte.

Aber warum musste es auf diesem Gebiet geschehen? Handelt es sich hier um das implizite Eingeständnis, dass die Objektivierung im Fall der moralischen Tatsachen auf ein unüberwindliches Hindernis stößt und die einzige Lösung darin besteht, die Wissenschaft, deren Regeln Durkheim formalisiert hatte, zu verlassen und zu der philosophischen Problematisierung zurückzukehren, die er hinter sich gelassen hatte? Wenn das zuträfe, wäre es unverständlich, dass man auch weiterhin von einer Bestimmung der Tatsache spricht. Das Verhältnis zwischen Soziologie und Philosophie, hier im Blick auf die soziale Tatsache, ist zweifellos subtiler. Wie wir sehen werden, kommt hier die Kohärenz der Durkheimschen Sichtweise ins Spiel, und das an einem mittleren Ort zwischen zwei Vorgehensweisen, die, soweit sie nicht deckungsgleich sind, ein besonderes Verständnis der moralischen Tatsache sichtbar werden lassen, nämlich als eine bis in ihre subjektive Dimension hinein soziale Tatsache. 


\section{Bestimmung einer Tatsache}

6 Der Text von 1906 muss offensichtlich vor dem Hintergrund des Vorworts zur ersten Auflage von Über soziale Arbeitsteilung gelesen werden, von dem Durkheim in der zweiten Auflage gut dreißig Seiten strich, »die uns heute überflüssig erscheinen«. In jener Passage versucht er, die soziologische Perspektive in ihrem Gegensatz zur klassischen Moralphilosophie sowohl Kantischer als auch empiristischer Prägung zu begründen. ${ }^{7} 1893$ bestreitet Durkheim, dass es bei der Erforschung moralischer Fragen die richtige Methode sei, ein allgemeines Sittengesetz zu formulieren, das letztlich doch nur die Projektion einer nicht wissenschaftlich konstruierten Vorstellung vom Menschen darstelle. Die »Wissenschaft der Moral« - einschließlich der von Renouvier 1869 veröffentlichten, die beträchtlichen Einfluss auf Durkheim ausübte - habe noch nicht $\mathrm{zu}$ ihren Grundlagen gefunden, weil sie einer deduktiven Methode verhaftet bleibe, die den Primat der Moraltheorie behauptet. Diese Methode, ganz gleich in welcher Form sie eingesetzt werde, sei ungültig, weil sie die Komplexität und Vielfalt der moralischen Tatsachen nicht zur Kenntnis nehme. Durkheim empfiehlt die Schaffung einer »Wissenschaft, die zunächst die moralischen Phänomene klassifiziert, die Voraussetzungen klärt, von denen die so gebildeten Typen jeweils abhängen, und schließlich deren Rolle bestimmt, also einer positiven Wissenschaft der Moral ${ }^{8}{ }^{8}$

7 Wenn solch eine Wissenschaft möglich sein soll, bedarf es jedoch eines stabilen Kriteriums, das definiert, was in die Taxonomie aufgenommen werden soll. Kurz, die Frage der Bestimmung der moralischen Tatsache oder auch der "moralischen Wirklichkeit" ist bereits gestellt - wobei klar ist, dass auch die "moralische Wirklichkeit« komplex und heterogen ist und zum Gegenstand einer Typologie werden muss, die deren Struktur erhellt. Und die Frage wird folgendermaßen beantwortet: Jede moralische Tatsache besteht aus einer auf diffuse Weise sanktionierten Verhaltensregel. Die Sanktion, verstanden als im Voraus festgelegte soziale Reaktion auf einen Regelverstoß, wird als das ausschlaggebende Kriterium für die Objektivierung der Tatsache dargestellt. Moral und Recht sind Spezies ein und desselben Genus, der Unterschied zwischen beiden kommt erst durch die Art der »Ausführung« der Sanktion herein, diffus im einen Fall, organisiert im anderen. Die Anwendung dieses Kriteriums macht den Vergleich zwischen Regel und Tatsache noch problematischer, da man ja die Frage nach dem subjektiv verteilten moralischen Urteil nicht in denselben Begriffen stellen kann wie in dem Fall, in dem sich eine sichtbare Institution, in der das Urteil formuliert wird, vollkommen klar abzeichnet.

Durch die Betonung der Sanktion erhebt man die Verpflichtung - und nicht die Tugend, wie Paul Janet in seiner Morale (1874) behauptete - zum Hauptmoment der Bestimmung der moralischen Tatsache. Hier zeichnet sich ein Element ab, das im Text von 1906 latent bleibt, ohne dadurch seine Bedeutung zu verlieren: Wenn die Pflicht in der Moral an erster Stelle steht, so in jener Form, die in der repressiven Sanktion erkennbar wird. ${ }^{9}$ Der Bezug auf die Repression ist 1906 gewiss nicht mehr aktuell. Dennoch ist es immer noch das Element der Sanktion, das als bestimmend gilt, insofern es innerhalb des allgemeinen Bereichs der Regeln moralische von rein technischen Regeln $\mathrm{zu}$ unterscheiden erlaubt. Anders gesagt, eine Handlung wird nicht einfach sanktioniert, wenn sie gegen eine Regel verstößt, sondern insofern sie gegen eine Regel verstößt. Die Sanktion ist keine Folge der Handlung, die analytisch in diesem Begriff enthalten wäre, sie ist vielmehr eine Folge des Verhältnisses zwischen der Handlung 
und der Regel, die auf dieses Verhältnis zurückgeht. Außerhalb ihres Verhältnisses zur Regel als Regel könnte solch eine Handlung niemals mit einer Folge vom Typ Sanktion verknüpft werden. Denn - so übersetzt Durkheim in seiner recht freien Interpretation der Kantischen Unterscheidung zwischen Vorschrift und Imperativ - das Verhältnis zwischen Sanktion und Handlung ist synthetischen Charakters. ${ }^{10}$ Nichts in der Handlung selbst macht sie für Sanktionen anfällig. Es bedarf einer Synthese zwischen Handlung und Sanktion, die, wie wir noch sehen werden, nur sozialer Natur sein kann und nach der man zu sagen vermag, dass ich bestraft werde, nicht weil ich dies, sondern insofern ich dies getan habe, das heißt aufgrund meines Verhältnisses zur Regel.

Wenn hier im Blick auf die Notwendigkeit einer Objektivierung der Tatsachen die Sanktion privilegiert wird, so bringt das eine Schwierigkeit mit sich, die das »Durkheimsche Dilemma« bereits erkennen lässt. Es geht hier darum, die Möglichkeit zu eröffnen, die Vielfalt der moralischen Tatsachen zu erfassen und zu ordnen, ohne auf die klassisch-philosophische Analyse des Begriffs des moralischen Handelns zurückzugreifen. Doch dieser Wechsel auf die Außenseite setzt gerade voraus, dass man sich auf die Wirkungen eines Verstoßes, auf die Folgen von Handlungen konzentriert, die der Moral widersprechen. Die Sanktion ist, wie Durkheim sagt, gerade deshalb ein Reagens, ein Mittel der Objektivierung, weil sie die Regel bezeichnet, insofern sie negiert wird. Bleibt zu klären, ob diese Verwendung des Reagens nicht eine unerträgliche Einschränkung bedeutet, weil sie, wie P. Pharo es ausdrückt, sämtliche Handlungen außer Acht lässt, in denen die Achtung vor einer Verpflichtung zum Ausdruck kommt, deren Missachtung keinerlei Sanktionsrisiko birgt. ${ }^{11}$

10 Allerdings kann der Einwand sich nicht mit diesem faktenbezogenen Hinweis begnügen. Denn Durkheims Argumentation entfaltet sich in der Begriffsökonomie des Verhältnisses zwischen Verpflichtung und Sanktion - es geht durchaus nicht darum, ob der Verstoß tatsächlich ein Risiko birgt, und auch nicht darum, ob der Handelnde dies weiß. Die Typologie der Sanktionen in Über soziale Arbeitsteilung dreht sich um diesen Punkt. Die repressive Sanktion zielt darauf ab, den Handelnden herabzusetzen. Sie besteht [im Französischen] ganz buchstäblich aus einem "Schmerz" (peine) - einer Strafe. Diese Zielsetzung hat nun aber erhebliche Folgen im Blick auf die Verpflichtung. Das Strafrecht - und die Moral unterscheidet sich nicht im Wesen davon, sondern nur in der Art, wie die Strafe erteilt wird - »sagt nicht, wie es das Zivilrecht tut: das ist die Pflicht, sondern sofort: Das ist die Strafe ${ }^{12}$, die Verpflichtung wird als immer schon bekannt vorausgesetzt. Die moralische Verpflichtung ist also durch eine spezielle kognitive Situation gekennzeichnet, die ihren Ausdruck in einer Sanktion bestimmten Typs findet. So wird auch ersichtlich, dass die Sanktionsperspektive eine Bewertung des moralischen Urteils keineswegs ausschließt, sondern sogar impliziert, wobei zu klären wäre, worin die charakteristischen Verpflichtungen der repressiven Sanktionen gründen, ob sie nun organisiert sind oder diffus.

\section{Pflicht und Begehren - mit Kant über Kant hinaus}

11 Wo hat die charakteristische Verpflichtung der moralischen Regeln ihren Ursprung? Zu dieser Frage steuert der Text von 1906 neue Elemente bei, indem er die klassische Kantische Argumentation, die ihm als Grundlage dient, abwandelt. Im Vergleich zum Kantianismus zeigt sich im Utilitarismus, insbesondere dem von Spencer, »eine vollkommene Unkenntnis des Wesens der Obligation«, da er die Folge eines Verstoßes 
nur analytisch als "mechanische Folge der Handlung « zu begreifen vermag..$^{13} \mathrm{Kurz}$ gesagt, die Unkenntnis basiert letztlich auf einem fehlenden Verständnis des Begriffs der Sanktion als synthetischer Verbindung zwischen Handlung und Regel. Die objektive Sicht der Sanktion - die ganz anders ist als bei einem Kantianer, setzt sie doch, wie Durkheim erkennt und fordert, eine "streng empirische Analyse« voraus - führt zu folgendem Schluss: Die Verpflichtung gründet nicht in der Natur des Gebotenen, sondern in der Tatsache, dass es geboten ist. Hinter den sanktionsbewehrten Verhaltensregeln, welche die moralische Verpflichtung verkörpern, muss man eine »besondere Autorität" sehen, kraft deren die Regeln »befolgt werden, weil sie gebieten «.$^{14}$ Letztlich erhält das von den repressiven Sanktionen aufgeworfene Problem damit eine Lösung: Die Verpflichtung muss gar nicht ausgesprochen werden, sie wird als immer schon bekannt vorausgesetzt, und deshalb, weil sie nicht ihrem Inhalt nach bekannt sein muss - weil man im Blick auf die moralische Verpflichtung nichts anderes $\mathrm{zu}$ wissen braucht, als dass sie verpflichtet, ein Wissen, das in ihrer Form als moralisches Gebot unmittelbar enthalten ist. Man könnte auch sagen, die Pflicht hat ihren Grund in sich selbst. Daher ihr Vorrang gegenüber dem Guten. Nicht in einem objektiv qualifizierten Guten, sondern in der Pflicht als »objektive Nötigung der Handlung", wie Kant sagt, muss die Moral begründet werden. Nach Kant muss das Gebieterische des Sittengesetzes, wie man sich erinnern wird, tatsächlich formal als Imperativ charakterisiert werden, der den »Willen als Willen« bestimmt, und nicht im Blick auf einen gewünschten Zweck. Es hat weder den subjektiven Charakter der Maxime noch den bedingten Charakter der Vorschriften, die $\mathrm{zu}$ den bloß hypothetischen Imperativen gehören. Es ist vielmehr kategorisch - wodurch nach Kant der Bereich der eigentlich praktischen Notwendigkeit definiert ist.

In eben dieser klassischen Form des Gebotes versucht Durkheim die moralische Tatsache in ihrer absoluten Besonderheit zu bestimmen. Aber sogleich verändert er dessen Bedeutung, indem er ein Prinzip formuliert, das nichts Kantisches mehr hat und von dem man auf den ersten Blick nicht versteht, wie man es mit der oben genannten Definition der formalen Verpflichtung vereinbaren könnte. Nach Durkheim ist der Pflichtbegriff selbst unzureichend und der reine Formalismus unhaltbar, denn »es ist unmöglich, eine Handlung nur deshalb zu vollziehen, weil sie geboten ist, ohne Rücksicht auf ihren Inhalt «. ${ }^{15}$ Die streng klassische Lösung ist nur teilweise richtig; sie muss um ein materielles Element ergänzt werden, das ihrer formalistischen Dimension zuwiderläuft. Den Vorrang der Pflicht gegenüber dem Guten - wobei das Gute für Kant »auch materialer, aber nur objektiver Bestimmungsgrund der Gegenstände der Handlung ${ }^{16}$ ist - kann man nur dann umstandslos behaupten, wenn man in der Pflicht selbst bereits ein Gut sieht und damit dem, was Kant für das rein formale Prinzip des Gesetzes oder Gebotes hält, einen Inhalt verleiht. Durkheim macht hier Folgendes: Er zeigt, dass ein genaues Verständnis des Pflichtbegriffs, eine Analyse der Pflicht nicht ohne das Gute auskommt und dass daher die Grundlagen einer nichtformalen Theorie der Moral gegen alle Erwartung innerhalb des strengen Rigorismus ${ }^{17} \mathrm{zu}$ finden sind.

Die Kritik des Kantischen Formalismus samt der Wiederaufnahme der Moral der Pflicht auf der Grundlage einer Universalisierung der Handlungsmaxime ist nichts Neues. Als Gemeinplatz der französischen Philosophie republikanischer Prägung, wie sie in der zweiten Hälfte des 19. Jahrhunderts gepflegt wurde, wandeln Autoren wie Barni, Renouvier, Fouillée oder Paul Janet sie in unterschiedlicher Weise ab. Die aufkommende Soziologie erneuert um die Jahrhundertwende diese Debatte und setzt 
eine der Kraftlinien fort: die Suche nach einer Triebfeder des Handelns, die den kategorischen Imperativ auf andere Weise in den affektiven Bereich einbringen könnte als die reine "Achtung fürs moralische Gesetz [...] als positive aber indirekte Wirkung desselben aufs Gefüh/ « ${ }^{18}$, die nicht ausreicht, um diesen subjektiven Bestimmungsgrund des Handels zu konstituieren, den die republikanische Moral durch geeignete Erziehungspraktiken reaktivieren möchte. Denn Universelles lässt sich nicht praktisch auf eine Abstraktion zurückführen und erst recht nicht auf die Formulierung eines Gebots, dessen bloße Form seine Geltung zu garantieren vermöchte. Frédéric Rauh fasste das Ziel 1890 in einem Buch zusammen, das für die Stellung des Problems der Moral in dieser Zeit von zentraler Bedeutung war: »Es gilt, Kants Logizismus durch die Rechtfertigung des mit der Idee versöhnten Gefühls zu überwinden. « ${ }^{19}$

In diesem allgemeinen Rahmen, in dem es darum geht, das Ziel des Universellen innerhalb der Affektivität zu finden und damit auf einer Ebene, die Kant in den Bereich des Pathologischen verweist, wird das Besondere an Durkheims Position dennoch rasch deutlich. Zunächst einmal, wie wir gesehen haben, in seiner Weigerung, sich auf dasselbe Diskussionsterrain zu begeben: Es geht um die Bestimmung der moralischen Tatsache, und das setzt voraus, dass man die moralische Wirklichkeit von außen betrachtet, nicht aus der Sicht der inneren Erfahrung des moralischen Subjekts und der Vorstellung, die das Individuum sich selbst von der Moral macht. Die Begründungsoder Wiederbegründungsbemühungen der Moralphilosophen, stark von Individualismus und Introspektion geprägt, können nicht gelingen (Kant, Renouvier, Janet, doch auch die englischen Utilitaristen verfallen dieser Kritik). Aber mehr noch: Durkheim will den Rigorismus nicht verbessern, indem er gewisse äußere Korrektive einbrächte, die ein Abgleiten in den Formalismus verhindern könnten, er möchte ihn noch verstärken. Seine Absicht ist es, ein von Kant und seinen Kritikern nicht bemerktes inneres Band zwischen der Pflicht und dem Guten, zwischen Verpflichtung und Begehren ausfindig zu machen. Die moralische Sache oder das Moralische (le moral) wie Durkheim es mit einer substantivierenden Wendung sagt, die einer Objektivierung und damit (gemäß der ersten Regel der Methode) der Wahrnehmungsweise einer außerhalb des Subjekts angesiedelten Sache entspricht - hat etwas objektiv Erstrebenswertes, womit der Begriff des Guten wieder in den Vordergrund gerückt und die Analyse der Pflicht verkompliziert ist. Das Paradoxon, das nur der Soziologe akzeptabel zu machen vermag, liegt darin, dass dieses "Erstrebenswertsein « nur einen Aspekt der Pflicht selbst darstellt, dass darin »etwas von der Natur der Pflicht « liegt. ${ }^{20}$

»Wir empfinden es als ein Vergnügen sui generis, unsere Pflicht zu tun, weil sie die Pflicht ist. ${ }^{21}$ Unter dem Vergnügen sui generis müssen wir ein Vergnügen verstehen, das sich nicht auf jenes Vergnügen reduzieren lässt, welches uns die Verfolgung von außerhalb der Moral liegenden Zwecken bereiten kann, ein Vergnügen, das durch die Pflicht selbst hervorgebracht wird, ein besonderes Vergnügen an der Tatsache, dass man einem moralischen Gebot unterliegt, das heißt dass man ihm als solchem gehorcht; ein Vergnügen an der "Bindung" - ein für Durkheims Analysen ganz zentraler Begriff, dessen beide Bedeutungen, die physische und die affektive, man hier nicht voneinander trennen darf..$^{22}$ Man ist in dem Maße "an etwas gebunden", wie man "durch es gebunden« ist. Das Vergnügen an der Bindung lässt sich nicht von dem Vergnügen trennen, gebunden, das heißt ganz buchstäblich: verpflichtet zu sein.

Der auf dieses spezielle Verständnis des Vergnügens reduzierte Eudämonismus ist zwar das Gegenteil des Rigorismus, koexistiert aber mit ihm und findet sogar Eingang darin. 
»Der Eudämonismus ist, wie auch sein Gegenteil, in der Sittlichkeit überall gegenwärtig. « $^{23}$ Durkheim erkennt sehr wohl, dass es sich hier um einen Widerspruch handelt, denn die Verpflichtung, die in der Sanktion, wie oben analysiert, zum Vorschein kommt, impliziert stets Strafe und Herabsetzung. Die Herausforderung, die sich dem Denken stellt, liegt nun darin, in dieser Herabsetzung eine Erhebung zu erkennen und sie mit einem authentischen Vergnügen ganz eigener Art zu verbinden. Anders gesagt, so offenkundig der Widerspruch auch sein mag, ist er doch nicht weniger konstitutiv für die moralische Tatsache als solche. Auch darf man sich nicht beeilen, ihn dialektisch aufzuheben, sondern muss sich bemühen, ihn zu verstehen, indem man die Analyse der Tatsachen vorantreibt.

Im Hintergrund hört man hier eine berühmte Passage aus Kants Kritik der praktischen Vernunft. Wie ist es möglich, eine Herabsetzung als Erhebung zu begreifen? Genau so findet die Frage sich bei Kant. Und der Hinweis auf die Achtung als positives Gefühl bringt dort die Lösung. Es gibt eine »Demütigung auf der sinnlichen Seite«, und es gilt zu verstehen, wie dem eine »Erhebung der moralischen, d. i. der praktischen Schätzung des Gesetzes« entsprechen kann, worin nun gerade die Achtung besteht, verstanden als positive Wirkung des Widerstands gegen die »Triebfedern der Sinnlichkeit«. Kants Antwort lautet: "Denn eine jede Verminderung der Hindernisse einer Tätigkeit ist Beförderung dieser Tätigkeit selbst. ${ }^{24}$ Mit der Folge, dass die Anerkennung des moralischen Gesetzes das Bewusstsein einer bestimmten Tätigkeit darstellt, die nichts anderes als die der praktischen Vernunft ist. Doch dieses Bewusstsein ist offenkundig nur indirekter Art. Es betätigt sich in der Aufhebung der Hindernisse, die die Sinnlichkeit dieser Tätigkeit entgegenstellt. Achtung resultiert aus einer Verdopplung der Hinderung: behindern, was das Gesetz behindert, das dann mit der Selbstbestimmung des Willens zusammenfällt. So verkehrt sich das Konzept der Demütigung bei Kant in sein Gegenteil: Was da gedemütigt wird, wird dies gerade durch die Achtung und bewegt sich im Bereich des Dünkels, das heißt unserer sinnlichen Neigung, es zu wagen, uns gegen das Gesetz zu erheben. So kann man denn - allerdings nur indirekt - sagen, der ausgeübte Zwang sei eine "Erhebung", die in einer "Selbstbilligung" besteht, und zwar jenseits jeglicher Lust, da "man sich dazu, ohne alles Interesse, bloß durchs Gesetz bestimmt erkennt « ${ }^{25}$

Durkheims Position ist hier radikaler und vor allem direkter. Wenn die Pflicht im Guten ist und umgekehrt, so weil Zwang und Herabsetzung wirklich in sich eine Erhebung darstellen - weil die Tatsache, dass man diese Herabsetzung lebt, etwas Erstrebenswertes hat. Nicht dass die Herabsetzung durch die Aufhebung eines Hindernisses gerechtfertigt würde, die eine andere, auf eine »intellektuelle Seite« des Subjekts als Subjekt des Gesetzes verwiesene Tätigkeit freisetzte, vielmehr ist diese Herabsetzung die Tätigkeit selbst, durch die man sich erhebt. Hier ist der Widerspruch besonders stark ausgeprägt, gewollt, betont, und man kann keineswegs hoffen, ihn durch den Rückgriff auf den Begriff des reinen Willens aufzulösen.

Allerdings handelt es sich um einen Widerspruch, der nirgendwo auf eine Auflösung des Subjekts, eine Aufspaltung zwischen Vernunft und Sinnlichkeit im Sinne zweier heterogener Gesichter der Subjektivität hinwiese. Kants großer Irrtum liegt für Durkheim gerade darin, dass er den Dualismus in dieser Weise verstand. Aber da das Gute in der Pflicht und die Pflicht im Guten ist, muss man im Gegenteil einräumen, dass Sinnlichkeit und Vernunft, ohne miteinander zu verschmelzen, in einem Punkt miteinander kommunizieren, der noch zu identifizieren bleibt. Die primäre Tatsache ist 
in der Tat die einer wechselseitigen Durchdringung der Elemente. Daraus folgt, dass man in der Moral jegliche Begründungsabsicht aufgibt. "Der Begriff des Guten erstreckt sich bis in den Begriff der Pflicht, so wie der Begriff der Pflicht und der Obligation sich bis in den des Guten erstreckt.« Kurz, wenn die wechselseitige Zusammengehörigkeit der Gegensätze real ist, muss man eingestehen, dass keinem der beiden Begriffe das Privileg einer ausschließlichen Begründung zukommt. Man darf kein Prioritätsverhältnis zwischen ihnen postulieren ${ }^{26}$, beide Begriffe müssen entschieden auf dieselbe Ebene gestellt werden. Daraus folgt nicht, dass die moralische Wirklichkeit sich in ihnen vollständig erschöpfte. Das moralische Leben besitzt zweifellos auch andere Merkmale, die durch eine empirische Analyse erhellt werden können. Die Moral variiert konstitutiv. Dieser "moralische Daltonismus», diese spezielle, in unendlich feinen Abstufungen variierende Färbung der Moral auf der Grundlage der beiden entgegengesetzten Farbtöne der Pflicht und des Guten, löst die phänomenale Wirklichkeit durchaus nicht in einem Relativismus bar jeglichen vereinheitlichen Prinzips auf, sondern rechtfertigt ein soziologisches Vorgehen, das diese Variationen auf der Basis eines einheitlichen Interpretationsschemas erfassen soll. So kann der Ersatz der These einer wechselseitigen Zusammengehörigkeit des Guten und der Pflicht für die der Begründung als Kritik an der Begründungsperspektive schlechthin gelten. Das Ziel des Vortrags von 1906 ist zugleich philosophischer und soziologischer Natur. Es geht darum, eine Tatsache zu bestimmen und dadurch in der Lage zu sein, die Tatsachen zu lesen und zu analysieren. Es geht nicht so sehr darum, die Moral zu begründen, als darum, sie zum Gegenstand einer Wissenschaft zu machen, die nur eine Sozialwissenschaft sein kann.

\section{Die Ambivalenz des Heiligen}

Die Besonderheit des Durkheimschen Verständnisses der Moral liegt also in der wechselseitigen Durchdringung der Gegensätze. Hier ist ein Widerspruch am Werk, den die Philosophie immer wieder reduziert oder dialektisch aufgehoben hat in ihrem Bemühen, die Moral unter Missachtung der Analyse der damit zusammenhängenden Tatsachen zu begründen. Die soziologische Sicht verfährt hier genau umgekehrt. Die Tatsache bestimmen heißt, den strukturierenden Charakter des Widerspruchs $\mathrm{zu}$ verstehen, heißt, die Tatsache zu begreifen, dass seine Termen ein polares Gefüge bilden, in dessen Inneren moralische Handlungen und Urteile ausgeführt und formuliert werden. Der religiöse Unterbau der Moral wird damit allerdings nur allzu sichtbar. In ihrem inneren Widerspruch erfasst, wird die moralische Tatsache auf das Element des Heiligen zurückgeführt, den Prüfstein der Durkheimschen Definition der Religion. Denn das Heilige, das sich Durkheim, wie er sagt, bei der Lektüre von Robert Smiths The Religion of Semites »offenbarte ${ }^{27}$, ist genau dies: eine bestimmten Entitäten zugeschriebene Qualität, die sie zum Objekt einer gleich starken anziehenden und abstoßenden Kraft macht, eines Begehrens und eines Verbots. Das Heilige ist daher durch seine »Ambivalenz« gekennzeichnet. Seine Definition geht von einer Negation aus: Es ist das, was nicht profan ist, was auf Abstand zum Profanen gehalten werden muss, was unter keinen Umständen damit vermengt werden darf. Zugleich aber ist es das, was das Profane anzieht, was dorthin strebt, ohne es jemals berühren zu können. ${ }^{28}$ Wenn es zutrifft, dass jegliche soziale Existenz eine Erfahrung des Heiligen voraussetzt, wobei die religiösen Tatsachen sämtlich diesem Definitionskern zugeschlagen werden, so bedeutet dies, dass auf einer bestimmten Ebene unserer sozialen Erfahrung das Gute 
und die Pflicht genau dasselbe sind und miteinander zu ein und derselben Sache verschmelzen. Das Heilige ist erstrebenswert, es ist sogar Objekt eines Begehrens ganz eigener Art, das sich nicht auf andere Formen sinnlichen Begehrens zurückführen lässt und dennoch unbestreitbar in der Sinnlichkeit verankert ist. Mit anderen Worten, und in Kantischer Sprache gesagt, das höchste Vermögen des Begehrens gehört in seinen Besonderungen doch stets dem Bereich der Affektivität an. Aber das Heilige ist auch verboten, es ist das Verbot schlechthin, das absolut »Abgesonderte«. Es weist im selben Maße in die Schranken, wie es erhebt; es erhebt im selben Maße, wie es Zwang ausübt. Man kann durchaus sagen, es sei Gegenstand von Achtung, sofern man nur hinzufügt, dass diese Achtung nichts mit dem nichtpathologischen und rein praktischen Gefühl zu tun, wie Kant es verstand. Bei Durkheim wird die Figur der Achtung mit ihrem zweifachen Kennzeichen des Erstrebens und der Herabsetzung über das Gefühl des Heiligen vollständig wieder in den Bereich der Sinnlichkeit integriert.

Dennoch bleibt das Heilige eine Tatsache. Es erhellt nicht nur die Koexistenz der Gegensätze, sondern ist deren Illustration und empirische Manifestation. Durch das Heilige finden die beiden Termen zu einer widersprüchlichen Einheit, aber der Widerspruch wird nicht aufgelöst. Ohne überwunden zu werden, zeigt sich der Widerspruch in einer einheitlichen Erfahrung, die die Moral unwiderruflich auf die Religion zurückführt. Aus dieser Radikalisierung der Analyse kann man den Schluss ziehen, dass am Grunde der Moral eine grundlegende und unausrottbare Religiosität zu finden ist. Die Moral kann sich durchaus säkularisieren, sie kann ihre theologische Grundlage verlieren, sich als menschlich und ausschließlich menschlich begreifen, aber sie setzt notwendig das Heilige voraus und bleibt in diesem Sinne stets religiös, ganz gleich, welche Entitäten sakralisiert werden. Durkheims Beitrag zur Idee des "säkularen Glaubens « (foi laïque), die in der Dritten Republik - vor allem von Ferdinand Buisson, Durkheims Vorgänger an der Sorbonne - vertreten wurde, präzisiert sich nicht im Sinne eines Überholens der Religion, sondern einer Verschiebung innerhalb einer moralischen Sphäre, die nun eine Sakralisierungsfunktion übernimmt, ohne die kollektives Leben nicht möglich wäre.

Was die moderne Manifestation des Heiligen unterscheidet und zugleich die Möglichkeit bietet, die besondere Kombination zwischen dem Guten und der Pflicht zu kennzeichnen, wie sie sich in den von Durkheim in Über soziale Arbeitsteilung durch den Begriff der organischen Solidarität definierten Gesellschaften findet, ist der Punkt, der im Zentrum des Heiligen liegt: die Tatsache, dass es der Existenz der sozialen Subjekte vollkommen immanent ist. Da es nicht mehr nach außen in eine theologische Transzendenz und nicht einmal in eine vergöttlichte Natur projiziert wird, ist es zum Individuum als solchem geworden oder genauer zur Person, dem späten Produkt eines historischen Individuierungsprozesses, den Durkheim in einigen Texten als "Individualismus « bezeichnet. ${ }^{29}$ Auf diesen Punkt konzentrieren sich die stärksten und entschiedensten sozialen Gefühle, die notwendig eine moralische oder strafrechtliche Reaktion erfordern. Das heißt aber auch, dass gerade das, was uns am stärksten verpflichtet - dem wir im Modus des strikten Gebots verpflichtet sind -, gerade das ist, was uns am nächsten liegt, so nahe, dass es mit uns selbst verschmilzt. So kann man sagen, das "Abgesonderte« - das heißt Heilige - sei zugleich das herausragende Objekt unserer »Sympathie « ${ }^{30}$, der Brennpunkt unseres unmittelbarsten Begehrens.

Die Verwendung des klassischen Begriffs der Sympathie darf uns nicht täuschen. Es handelt sich keineswegs um eine Rückkehr zur angloschottischen Tradition, in der 
dieser Begriff erstmals abgegrenzt wurde. Bei Durkheim bezeichnet "Sympathie« zwar durchaus eine Gestalt der Sinnlichkeit, doch in dieser eigenständigen Form vermag das Begehren nach dem Gesetz, das dem Gesetz selbst innwohnende Begehren, sich zu zeigen. Es ist keine Sympathie für die andere und als solche wahrgenommene Person und auch keine Sympathie, die einen Selbstbezug maskierte und auf eine in sich selbst zurückgebogenen Bahn verwiese, sondern ein auf die Person als solche gerichtetes Begehren, das heißt auf die Form des Individuums, die in jedem von uns gleichermaßen verkörpert ist. Kurz, das Heilige kommt dem Subjekt zwar näher als jemals zuvor, bleibt aber dennoch im Bereich des Abgesonderten. Die persönliche und menschliche Wendung der Moral in den modernen Gesellschaften muss daher mit Hilfe des oben beschriebenen Schemas interpretiert werden. Darin aktualisiert sich ein bemerkenswertes Gleichgewicht, ein rechtes Verhältnis zwischen der Pflicht und dem Guten, denn das Objekt der Pflicht erhellt besser als alles andere, dass dieses Objekt tatsächlich ein Gut ist, nämlich ein Gut für uns. Hier stellt sich allerdings die Frage, welche Bedeutung man dem "wir« zu geben hat. Damit treibt man die Analyse der wechselseitigen Durchdringung der Gegensätze einen Schritt weiter, ohne die das Moralische jegliche Konsistenz verlöre; man verlässt den Bereich des Heiligen, der eine Wirklichkeit stets nur enthüllt, sie aber nicht begründet, und richtet das Augenmerk auf die Struktur des moralischen Urteils.

Durkheim kommt darauf in seinem Werk immer wieder zurück. Das Individuum ist zu dem geworden, was in den modernen Gesellschaften für uns den größten Wert besitzt. Dabei ist allerdings zu bedenken, und hier liegt der unwiderrufliche Unterschied zu den liberalen Thesen, dass es diesen Wert dennoch nicht durch sich selbst besitzt. Es hat ihn vielmehr schrittweise in einem sozial determinierten Individuierungsprozess erlangt und sozial determiniert heißt nicht bloß, gewissen sozialen Bedingungen unterworfen, sondern gänzlich von einer sozialen Dynamik gesteuert, deren autonome Bewegung dazu drängt, die Existenz eines ständigen übergeordneten Akteurs zu postulieren, den man nicht anders denn als Gesellschaft bezeichnen kann.

Genau an diesem Punkt setzt die Soziologie sich der Kritik aus. Die »kollektive Persönlichkeit" wird - in derselben Weise wie der Begriff des »kollektiven Bewusstseins", der geschaffen wurde, um das Problem der von der Gruppe als solcher produzierten Vorstellungen zu lösen - zur höchsten Quelle erhoben, aus der die Sittlichkeit fließt, welche die Individuen in der jeweiligen moralischen Situation übernehmen. Ist sie etwas anderes als eine Abstraktion, eine weitere ideelle Entität? Man begreift durchaus, dass die Verwendung eines so umstrittenen Begriffs, der gerade jene Art metaphysischer Fiktion wiedereinzuführen scheint, die Durkheim bei seinen Vorgängern ablehnte (bei Comte etwa, bei dem die Menschheit eben genau die Figur eines Großsubjekts darstellte), im vorliegenden Fall eine gewisse Berechtigung haben könnte. Tatsächlich ist die hier postulierte Kollektivpersönlichkeit vor allem das Objekt eines Begehrens. Es ist jenes an sich begehrenswerte Gut, das einen der für das moralische Element konstitutiven Pole belegte. Der Begriff ist eine Reaktion auf zwei Erfordernisse: $\mathrm{Er}$ markiert durch das individuelle subjekt eine bestimmte Gemeinschaft, ohne die man nicht verstehen könnte, dass der moralische Zweck wirklich ein Gut darstellt und tatsächlich das subjektive Begehren aktiviert. Und er verweist zugleich auf einen qualitativen Unterschied, ohne den sich die Dimension der Verpflichtung ihrerseits nicht herleiten ließe. Kurz, der angestrebte Zweck muss 
zugleich derselbe und ein anderer sein, er muss den Individualitäten, die ihn anerkennen, zugleich immanent und transzendent sein.

Die Figur des Kollektivsubjekts trägt also Spuren der Ambivalenz des Heiligen, bis auf den Unterschied, dass die philosophische Argumentation theoretisch zu konstruieren vermag, was die Religionssoziologie nur feststellen kann. Eine bemerkenswerte Situation: Hier überholt die Philosophie die Soziologie, und dies unter Durkheims eigener Feder. Gewiss, die Argumentation basiert auf dem Postulat der Nichtrückführbarkeit des Ganzen auf seine Teile und des qualitativen Unterschieds zwischen beidem. Doch hier wäre zu präzisieren, dass Durkheim auf dieses Postulat nur als Lösung für ein theoretisches Dilemma zurückgreift, das Dilemma der Koexistenz der Gegensätze in ein und derselben Entität, dem Moralischen, das eine bestimmte Art von Tatsachen umfasst. Wenn der innere Widerspruch sich auf dieser Ebene als strukturierend erweisen kann, so weil die Gesellschaft jenseits ihrer Teile durchaus eine Person darstellt. Und mehr noch weil die Heiligkeit, die man jeder Person formell zugesteht, aus jener Quelle des Heiligen stammt, die die Gesellschaft darstellt - aber dann muss man eben auch sagen: die Gesellschaft als Person.

Das Postulat ist also nicht willkürlich. Aber ist es auch notwendig? Streng genommen muss man sagen, wenn man sich an das Problem hält, wie es sich rein theoretisch stellt, sind zwei Lösungen zulässig: die Gesellschaft und Gott. Durkheim erkennt das und entzieht sich dem nicht: Zwischen beiden "muß man wählen «. ${ }^{31}$ Menschliche oder göttliche Synthese, beides ist vorstellbar, und man erkennt am Ende, dass das von Kant formulierte Postulat der praktischen Vernunft in gewissem Maße wieder zu seinem Recht kommt. Aber vor allem erkennt man den Einfluss Auguste Comtes: Nur zwei Stadien können als systematisch gelten, das theologische und das positive, und die lange kritische Übergangsphase vom einen zum anderen, die Comte als das metaphysische Stadium bezeichnete, diente vor allem dazu, den synthetischen Pol ins Innere der Welt zu verlegen. Diesen Pol, der es tatsächlich erlaubt, eine positive, nichttheologische Moral zu begründen (während es keine kritische oder metaphysische Moral gibt), identifizierte Comte selbst ganz unmittelbar mit der Menschheit. Dennoch tun Comte und Durkheim im Wesentlichen dasselbe. Sie lokalisieren den höchsten Zweck menschlichen Tuns im Diesseits, ohne deshalb auf eine gewisse Transzendenz zu verzichten. Und wenn Durkheim sagt, die Wahl zwischen den beiden Postulaten sei ihm gleichgültig - was allerdings der zuvor ausgesprochenen Aufforderung zu dieser Wahlentscheidung $\mathrm{zu}$ widersprechen scheint -, ist dies so $\mathrm{zu}$ deuten, dass die soziologisch begründete positive oder auch soziale Moral zwar keineswegs alle Spuren von Religiosität verloren hat, aber deren aktives Prinzip auf anderen Wegen als denen des theologischen Denkens wiederfinden möchte.

Es bleibt indessen, dass diese Sicht der Moral untrennbar mit einer bestimmten Konstellation der Gegenwart verbunden ist, jener nämlich, in der »das Ideal der Gesellschaft eine besondere Form des menschlichen Ideals ist « $^{32}$ und der Wert für die Gruppe und der Wert für mich innerhalb des sozial und historisch konstituierten Begriffs der Person in ein direktes Verhältnis zueinander treten. Allerdings hüte man sich hier vor einem Irrtum: Was da als Wert für mich bezeichnet wird, ist kein rein individueller und partikularisierter Zweck. Es gibt hier keine utilitaristische Regression, keine Anleihe bei der Darstellung der moralischen Evolution nach Spencer, dem Bannerträger des liberalen Fortschritts, für den die Gesellschaft nur ein Mittel zur Verfolgung individueller Zwecke ist. Für Durkheim ist die Sakralisierung des 
Individuums in Wirklichkeit eine Sakralisierung der Gesellschaft in ihm, der Gesellschaft in individuierter Form. Daher handelt es sich um einen Wert, der im Individuum über das Individuum hinausgeht: einen Wert, in dem das Individuum sich und die anderen aus einer Perspektive wahrnimmt, die die empirischen Individualitäten transzendiert und sie zum Kollektivwesen als solchem integriert.

\section{Das Ideal der Gesellschaft, die sich selbst will}

Durkheims Soziologie der Moral, darauf sei hier noch einmal hingewiesen, ist eine situierte Soziologie. Die Vielfalt der moralischen Tatsachen in ihren unterschiedlichen sozialen Formen lässt sich nur auf der Grundlage einer Grammatik des Guten und der Pflicht entziffern, deren Klärung, wie wir gesehen haben, ihrerseits von einem bestimmten Verständnis der modernen Gegenwart als historischer Aktualisierung eines bestimmten konzeptionellen Gleichgewichts abhängt. Damit sind wir unmerklich von einer Bestimmung der moralischen Tatsache zur Einschätzung einer bestimmten Art von Moral übergegangen, der unsrigen nämlich. Die Verbindung zwischen Gesellschaft und Individuum erfolgt dort auf überaus paradoxe Weise. Einerseits war die Gesellschaft dem individuellen Bewusstsein noch nie so immanent, weil sie ihm unmittelbar in Gestalt eines Gutes erscheint, dessen Prüfstein der Begriff der Person darstellt, eine reine Form, die in den empirischen Individualitäten nicht aufgeht. Aber andererseits tritt auch ihre Transzendenz hervor, denn das moralische Ideal der Person impliziert eine Überschreitung. So eng wie noch nie zuvor miteinander verbunden und auf denselben Punkt fokussiert, widersprechen das Gute und die Pflicht einander unablässig, oder besser gesagt, der Widerspruch wird in seiner ganzen Radikalität wahrgenommen, weil er die Struktur der moralischen Tatsache definiert, sodass die Pflicht und das Gute einander schon aufgrund der Bewegung widersprechen, durch die jene dieses verwirklicht.

Man erinnere sich an die Entwicklung, die Durkheim in Über soziale Arbeitsteilung beschreibt. Da die primitive Gruppe eine relativ einfache Struktur besitzt, drängen die kollektiven Vorstellungen sich dort auf, weil sie sich aufdrängen können: Die Gesellschaft ist in ihrer Gänze vorstellbar, und das Individuum denkt sich nur im Verhältnis zu ihr und nur in dem Maße, wie es sie denken kann. Einzelbewusstsein und Kollektivbewusstsein sind deckungsgleich, und Ersteres ist im Vergleich zu Letzterem nur von geringer Bedeutung, eben weil das Kollektiv individuell vorstellbar ist. Umgekehrt gilt, »je weiter wir in der Geschichte fortschreiten, desto riesenhafter und komplexer wird die menschliche Zivilisation; je weiter sie daher das Bewußtsein des Einzelnen übersteigt, desto stärker empfindet das Individuum die Gesellschaft als ihm selbst gegenüber transzendent ${ }^{33}$ Diese Situation entspricht zunächst einer funktionalen Differenzierung, die jedem Subjekt eine bestimmte Besonderheit zuweist, deren Bedeutung von einem für ihn nicht direkt einsehbaren letzten Zweck abhängt. Diese Differenzierung ist zwar Ausdruck einer bestimmten Form gesellschaftlichen Zusammenhalts, der organischen nämlich, aber die Gesellschaft bleibt uns in einem anderen Modus »innerlich $\aleph^{34}$ als dem der souveränen kollektiven Vorstellungen. Dieser Modus ist derjenige der Figur der Individualität, die in zweifachem Sinne eine gesellschaftliche Hervorbringung darstellt, nämlich insofern die Gesellschaft sie hervorbringt und insofern sie es der Gesellschaft ermöglich, sich trotz der Aufspaltung in Einzelbewusstseine dauerhaft als kohärente Totalität zu erschaffen. 
Das Paradoxon sei hier noch einmal verdeutlicht: Die Gesellschaft ist in uns in Gestalt des Individuums präsent, das sich auf den ersten Blick als nichtsozial gibt. ${ }^{35} \mathrm{Aber}$ sie ist auch insofern präsent, als dieses Individuum nicht mit unserer Individualität identisch ist oder zumindest als diese Individualität im Innern als verdoppelt erscheint. Damit wird deutlich, dass die soziologische Sicht nicht so sehr eine Neudefinition des Verhältnisses zwischen Individuum und Gesellschaft ist, sondern vielmehr die schwierige Konzeptualisierung eines anderen Begriffs des Individuums, als der traditionelle individualistische Ansatz ihn bietet. Durch das Individuum, in seiner doppelten Modalität verstanden, existiert die Gesellschaft als differenzierte Einheit innerhalb einer komplexen Situation, in der individuelles und kollektives Bewusstsein unvermeidlich voneinander getrennt sind. Durkheim gelangt auf diese Weise zu einem Dualismus ganz eigener Art: einem Dualismus nicht des Individuums und der Gruppe, sondern einem in die Individualität selbst eingelassenen Dualismus zwischen der durch die Annahme der Kategorie der Person bestätigten Präsenz des Sozialen und der für sich rein empirischen Präsenz der je besonderen Individualität. ${ }^{36}$

Die Koexistenz der Gegensätze oder genauer: der strukturierende Charakter des Widerspruchs in der Moral - die Tatsache, dass keiner der beiden konstitutiven Termen den Vorrang besitzt und keine vereinheitlichende Synthese zulässig ist - verweist auf eine bestimmte Bedeutung des Begriffs »Ideal«. Durkheims Ideal oder »idealer Typus« hat nichts mit Webers Idealtypus zu tun. Es handelt sich nicht um ein theoretisches Konstrukt, mit dessen Hilfe der Soziologe die konkreten Tatsachen im Blick auf ihre Abstände ordnet, wobei er sich vor Werturteilen hütet. Der ideale Typus ist dagegen ein normatives Konzept und bezieht sich auf das, was die Gesellschaft als Zweck hervorbringt, was für das Individuum moralisch erstrebenswert ist, als Selbstüberschreitung, die mit ihrer Verwirklichung zusammenfällt. Mit dem Ideal sind wir durch jene Form von affektivem Band verbunden, die nur das moralisch Gute hervorzubringen vermag. Doch dadurch werden wir zugleich von einem Teil unsererselbst getrennt, aufgrund der sinnlich-empirischen Individualität. Dass die Gesellschaft das Ideal hervorbringt, hat in diesem Kontext eine ganz präzise Bedeutung: Es heißt nämlich, dass sie Vorstellungen hervorbringt, die im individuellen Bewusstsein die Rolle eines Pols spielen, der zugleich anzieht und als Instanz normativer Gebote fungiert. Auf der Höhe des Ideals zu sein, das wir als Mitglieder derselben Gruppe teilen, übersetzt sich unweigerlich in ein Begehren und eine Verpflichtung. Daraus wird deutlich, dass der Begriff des Ideals ein bestimmtes Verständnis des Werts impliziert und zugleich eine Antwort auf den häufig gegen Durkheims Objektivismus vorgebrachten Einwand liefert, er bleibe in der konformistischen Moral gefangen, da er sie von tatsächlich existierenden, in externen Normen verkörperten sozialen Faktoren abhängig mache, die nur mit Sanktionen arbeiten. Das Ideal ist nicht die Gesellschaft, wie sie unter den aktuellen Bedingungen der Gruppenstruktur (morphologie), des Zustands der Kommunikationswege, der Determinanten des internen sozialen Milieus existiert $^{37}$, es ist vielmehr die Gesellschaft, die auf der Grundlage dieses Substrats sich selbst in unseren eigenen Handlungen will, insofern diese sich auf ihrer eigenen Ebene als zugänglich für eine moralische Regulierung erweisen. Die interne Korrelation des Guten und der Pflicht führt zu Folgendem: Wir erkennen, dass wir, indem wir das Ideal wollen, insgesamt darin übereinstimmen, dass die Gesellschaft sich in uns will, das heißt auch: sich durch uns verwandelt. So wird verständlich, dass die »wahre Natur der Gesellschaft « ${ }^{38}$, wie Durkheim als Antwort auf Darlu sagt, sehr wohl gegen einen bestimmten Zustand der geltenden Regeln revoltieren kann, falls sich herausstellt, dass 
diese Regeln sich nicht mehr im Einklang mit dem Ideal befinden, nach dem die Gesellschaft sich will. In diesem Fall ist die Revolte und nicht der Konformismus moralisch. Und dies aufgrund einer Notwendigkeit, die sich allen auf der Ebene des Handelns als des realen Modus der Selbsterzeugung der Gesellschaft ergibt.

\section{Werturteile}

33 Hier zeichnen sich nun die Mittel zur Überwindung des "Durkheimschen Dilemmas« ab. Dieses Dilemma ist in der Tat nur dann unlösbar, wenn man bei einem Gegensatz zwischen äußeren Normen und Subjekt verharrt und das Problem der Moral als ein Problem der Einverleibung begreift, wobei man die Arbeit am Begriff der Individualität übersieht, der die Grundlage der Theorie bildet. Die Art, wie die Kantische Position übernommen und zugleich umgangen wird, auf der Grundlage der zentralen These einer wechselseitigen Zusammengehörigkeit der Gegensätze innerhalb der moralischen Tatsache und ihrer Abwandlung im Begriff des Ideals, lässt ein anderes Gesicht der Durkheimschen Soziologie aufscheinen, das frei von jenem Objektivismus und Konformismus ist, die man ihr gewöhnlich zuschreibt.

Dieses andere Gesicht zeigt sich am ausgeprägtesten in Durkheims Behandlung der "Werturteile« in seinem Vortrag auf dem Internationalen philosophischen Kongress in Bologna 1911. ${ }^{39}$ Auch dort wird das Problem Kantisch formuliert: Wie sind Werturteile möglich? Im klassischen Rahmen einer Analytik des Urteils stellt die Frage nach dem Wert in der Tat eine Klippe dar. Während Wirklichkeitsurteile lediglich »sagen, was ist«, treffen Werturteile eine Aussage über den Wert, den Dinge "für ein bewusstes Subjekt besitzen . $^{40}$ Wie soll man nun dieses Verhältnis und die damit verbundene subjektive Begründung verstehen, wenn es zutrifft, dass Werte objektiv gelten und Werturteile daher ihre eigene Objektivität besitzen?

An sich sind Empfindungsurteile - was das Subjekt empfindet - nur eine bestimmte Art von Wirklichkeitsurteilen. In diese Kategorie fallen etwa Geschmacksurteile oder „Vorlieben«, wie Durkheim sie nennt. Werturteile haben dagegen einen »objektiven Charakter « ${ }^{41}$ ganz eigener Art, weil sie den Wert der Sache betreffen. Die Sinnlichkeit des Subjekts, in der sich eine Vorliebe abzeichnet, bringt daher nicht das Urteil hervor. Beschränkt sie sich dann darauf, Eigenschaften eines Dings zur Kenntnis zu nehmen und etwas zu erkennen, das außerhalb ihrer selbst existiert und sich ihr aufdrängt? Und grundsätzlicher noch: Was bedeutet das Wort "Ding ", wenn dieses mit einem Wert ausgestattet ist? Durkheims Antwort erfolgt über eine Analyse der Bewertung, die er von der Vorliebe unterscheidet. Darin scheint das Subjekt eine Dimension des Objekts zur Kenntnis zu nehmen, die unabhängig davon ist, wie es das Objekt wahrnimmt. Doch auch wenn der Wert des Objekts unabhängig von seinem Verhältnis zu dem wertenden Subjekt ist, setzt er dennoch in seiner Definition die Setzung solch eines Verhältnisses voraus. Der Wert existiert »in gewissem Sinn $\aleph^{42}$ außerhalb meiner selbst, aber nur in gewissem Sinn. Die Einschränkung ist entscheidend. Das Werturteil ist kein Wirklichkeitsurteil, weil der Wert nicht in derselben Weise existiert wie ein Sachverhalt, und dennoch existiert es außerhalb des Subjekts, objektiv. Zwischen dem Ding und dem Sachverhalt verläuft eine Grenze, die Durkheim zu stabilisieren versucht. Der Wert liegt durchaus in dem Ding. Für diesen objektiven oder sachlichen Charakter gibt es zwei Indizien: Das Werturteil ist grundsätzlich kommunizierbar; und es ist begründbar, es lässt sich nur bestätigen, wenn man es mit unpersönlichen Gründen untermauert. Beim 
Akt des Begründens - und damit antizipiert Durkheim in befriedigender Weise ein wichtiges Problem der zeitgenössischen handlungsorientierten Soziologie, sofern man sich nicht darauf versteift, ihn für einen Gefangenen seines "Dilemmas« zu halten ${ }^{43}$ betätigt sich das Subjekt in einem unpersönlichen Modus.

Die Frage lässt sich also folgendermaßen präzisieren: Wie lassen sich jene beiden Merkmale erklären, die Unabhängigkeit von gefühlsmäßigen subjektiven Bestimmungen und das Verhältnis zum wertenden Subjekt und damit die Implikation einer bestimmten Form von Gefühlen? Oder auch: „Wie kann ein Gefühlszustand unabhängig sein von dem Subjekt, das ihn erfährt? ${ }^{44}$ Bei dieser Frage geht es nicht allein darum, die Existenz einer verkannten Dimension des Innenlebens aufzuweisen, die sich nicht allein mit den subjektiven Ressourcen erklären lässt und sich dem Subjekt in einem Modus aufdrängt, der dennoch mit ihm verbunden bleibt - dem des Gefühls nämlich. Es geht auch darum, einen neuen Blick auf die Dinge zu werfen, nach Maßgabe dieses Gefühls, das sich mit den Dingen vermengt, und sie gewissermaßen "anzureichern ${ }^{45}$ In diesem Sinne ist der »Gefühlszustand « der Wert des Dings, etwas Äußeres, das auf derselben Ebene liegt wie das Ding, zu dem er hinzutritt. Im buchstäblichen Sinne ist es die auctoritas gewisser Dinge in ihrem Dingsein, die hier ins Spiel kommt. ${ }^{46}$

Die Frage nach der Möglichkeit der Werturteile verschiebt, auf die Logik der Wertung zurückgeführt, das Gravitationszentrum der Frage nach der Moral. Es geht nicht mehr nur darum, die moralische Bewertung im Sinne eines Urteils über Handlungen, sondern auch und vor allem Urteile über Dinge zu betrachten, die mehr sind als Dinge oder zumindest von der Art jener sozialen Dinge, die das Durkheimsche Denken zu erfassen versucht: Dinge, deren Realität im strengen Sinne - Status der res - ihre eigene Wahrnehmung dadurch einschließt, dass sie sich dieser Wahrnehmung aufzwingt. Die Beschreibung ihres Konstitutionsprozesses setzt in erster Linie voraus, dass man von einer summarischen Verdinglichung, das heißt einer reinen Objektivierung Abstand nimmt. Denn damit führte man den Wert lediglich auf einen Zustand innerhalb des Subjekts zurück, der durch die inneren Eigenschaften einer bestimmten Art von Objekt ausgelöst wurde. Über den Wert urteilen hieße dann einfach, die Wirkung einer bestimmten objektiven Realität im Subjekt zur Kenntnis zu nehmen, den Weg, der im Sinne eines Kausalvorgangs von außen nach innen führt. Eine falsche Perspektive, die darauf hinausläuft, den Dingen Eigenschaften an sich zuzuschreiben, durch die sie in der Lage wären, einen - günstigen oder ungünstigen - subjektiven Zustand herbeizuführen, der seinerseits ein ihn betreffendes positives oder negatives Urteil auslöste. Die Existenz eines objektiv Guten wird zugestanden, aber nur um den Preis einer zweifachen Verabsolutierung: Verabsolutierung der Eigenschaften der bewerteten Sache und Verabsolutierung bestimmter Eigenschaften des wertenden Subjekts, das von diesen Eigenschaften affiziert wird. Dieses Subjekt könnte man sich in zweierlei Weise vorstellen: Entweder nimmt man das Individuum als Grundlage und kehrt zu einer "psychologischen Theorie der Werte« zurück, oder man weist den Primat der Gruppe als solcher zu, verstanden als mit gewissen stabilen Eigenschaften ausgestattetes Großsubjekt, ganz gleich, in welchen Formen es sich historisch verkörpern mag. Es liegt auf der Hand, dass keine dieser Lösungen befriedigen kann. Vor allem durch die Ablehnung der zweiten Lösung wird die Durkheimsche Sicht deutlicher. 1911 hat die Kategorie des »Kollektivsubjekts« - im Unterschied zu dem, was man im Text von 1906 erkennt - eine eindeutig polemische Bedeutung. Kann die 
Bewertung allein deshalb schon als objektiv bezeichnet werden, "weil sie eine kollektive ist «? ${ }^{47}$ Alles hängt hier vom Verständnis dieses Adjektivs ab. Denn die Klippe droht im Grunde dieselbe zu sein wie in der psychologisierend-individualistischen Theorie: Wir haben immer noch keine Möglichkeit, zu erfassen, dass die Werte variieren, ja dass sie gleichsam konstitutiv variieren, wie man sagen könnte. Führt man die Werte auf einen einheitlichen Bewertungspol zurück, der einer wesenhaften Charakteristik des Subjekts entsprechen soll, für das dieser Wert wertvoll ist, verflüchtigt sich die Realität der Werte, nämlich ihre Vielfalt. Beim Individualismus stellt sich das Problem im Blick auf die Unmöglichkeit, jegliche subjektive Wahrnehmung auf ein und denselben Typus zurückzuführen - in dem letztlich eine ebenso abstrakte Bestimmung der Wertschätzung des "Lebens« im biologischen Sinne des Wortes und unabhängig von jeder weiteren Spezifizierung vorherrschte..$^{48}$ Auf der kollektiven Ebene geht es vielmehr um die Betonung der Unmöglichkeit, innerhalb der Gesellschaft - verstanden als Großsubjekt, das sich nicht auf die einzelnen Subjekte reduzieren lässt, aus denen es besteht - ein fundamentales Bewertungsprinzip zu finden, auf das alle Werte letzten Endes zurückgeführt werden könnten. Vergeblich verweist man hier auf die Erfordernisse von etwas, das von der Art des »sozialen Lebens" wäre. Denn diese Bestimmung wäre allzu vage, um einen Wert $\mathrm{zu}$ konstituieren, der alle sozialen Werte zu einer Einheit zusammenfassen könnte, zumindest solange als man nicht versucht hat, diesen Begriff zu präzisieren.

Ein wesentliches Merkmal der Durkheimschen These ist die Distanz zu einem biologischen Vitalismus. Das Kollektivsubjekt ist kein Großorganismus, dessen Funktionen eine Lebensordnung definierten, mit der alle Aktivitäten konform gehen müssten und die nach dem Maßstab solch einer Konformität bewertet werden könnten. Der Begriff der sozialen Nützlichkeit, der Aufrechterhaltung oder Förderung des Lebens der Gruppe als Gruppe, vermag kein einheitliches Bewertungskriterium zu definieren. Zum Ausdruck kommt darin zunächst einmal die Vielfalt der sozialen Werte, ihre Heterogenität, ihre Inkommensurabilität (ökonomische, moralische, ästhetische, wissenschaftliche Werte), aber vor allem die Unmöglichkeit, in all den dadurch definierten Tätigkeitsbereichen den Vorrang des Nützlichkeitskriteriums zu belegen ${ }^{49}$ : "Das Leben, so wie es die Menschen seit jeher aufgefaßt haben, besteht eben nicht nur darin, das Budget des individuellen oder sozialen Organismus exakt festzulegen, mit möglichst geringem Aufwand auf äußere Reize zu reagieren, die Ausgaben genau nach den Einnahmen zu bemessen. Leben heißt vor allem handeln, handeln ohne $\mathrm{zu}$ rechnen, einfach aus Freude am Handeln. $\aleph^{50}$

Die Definition lässt die Triebfeder der Durkheimschen Konzeption des Wertes erkennen und zeigt, dass die Distanz gegenüber einer vitalistischen Position, die sich an ein organisch-funktionales Verständnis des Kollektivs anlehnt, in Wirklichkeit der Übernahme der Handlungsperspektive untergeordnet ist. Soziales Leben ist Handeln und mehr noch eine Vielzahl unterschiedlicher Handlungen, die sich nicht auf eine einzige Zwecksetzung zurückführen lassen. Gewiss, die Gesellschaft will ihren Fortbestand sichern. Doch die Sicherung des Fortbestands heißt für sie nicht, eine bestimmte vitale Funktionsweise gleichförmig zu reproduzieren, auf Dauer zu stellen und zu stabilisieren. Den Fortbestand sichern heißt vielmehr, sich zu verändern und eine Bewegung abzuwandeln, deren bloße Reproduktion den Zusammenbruch bedeuten müsste. Denn das Handeln der Individuen auf den diversen Wegen, die sie einschlagen mögen, ist der einzige Inhalt, den man dem Begriff des »sozialen Lebens" zu geben vermöchte. ${ }^{51}$ Das soziale Leben ist nicht das Leben des Gesellschaftskörpers im 
Sinne eines Organismus übergeordneter Art; es ist die Gesamtheit der individuellen Handlungen, durch welche die Gesellschaft wirklich lebt, sich ständig erneuert und sich dadurch verändert. Das einzige Prinzip, auf das man den Wertbegriff in seiner unmöglich zu erfassenden Differenzierung zurückführen könnte, ist das der Handlung. Solch ein Prinzip schließt aber aus, dass man den Dingen selbst aufgrund innerer Eigenschaften die Qualität zuschreiben könnte, die sie als Werte definiert. Diese Qualität wird ihnen vielmehr notwendig hinzugefügt durch die Art und Weise, wie das Subjekt sich in seinem Handeln auf sie bezieht.

\section{Gesellschaftliches Handeln und handelnde Gesellschaft}

Nun gilt es natürlich noch die Natur dieser Hinzufügung, dieser im Handeln erfolgenden Anreicherung der Dinge zu verstehen. Welcher Zusammenhang besteht zwischen Handlung und Bewertung? Wenn der Wert nicht in dem Sinne in den Dingen liegt, dass sie in sich gewisse Qualitäten besäßen, die das Subjekt dazu bestimmen, sie als mit Wert ausgestattet anzuerkennen, muss man dann nicht sagen, dass die Erfahrung der Werte nicht als Erfahrung existiert, sondern dem Bereich einer rein intellektuellen Erfassung einer außerhalb jeglicher Erfahrung liegenden Realität oder kurz: einer noumenalen Realität angehört? Hier zeichnet sich die Kantische Alternative ab. Sie wird indessen aus einem Grunde zurückgewiesen, der sich aus der oben beschriebenen Definition des Ideals und seines Verhältnisses zum Handeln ergibt. Die Kantische Sicht führt zu einem versteinerten und hypostasierten Verständnis des Ideals, das mit der ihm zugestandenen Variabilität unvereinbar ist. ${ }^{52}$ Und dabei geht es nicht nur um die Tatsache, dass das Ideal historisch und sozial variiert. Das Argument verweist nicht einfach nur auf den durch sozialgeschichtliche Analysen bestätigten Pluralismus der Wertungsprozesse. Es sieht die Variation vielmehr in der "Natur der Dinge« angelegt und rechnet das Ideal folglich den natürlichen Phänomenen und ihrer Bewegung zu. Auf dieser Ebene klärt sich der Vorrang der Handlungsperspektive. Wenn man das Ideal außerhalb der Natur setzt, trennt man es von der Handlung, in der sich das Leben der Subjekte bestimmt, die ihr Verhalten an den Werten ausrichten. Oder sagen wir es anders: Wenn man Werturteile verstehen will, darf man sie gewiss nicht den Wirklichkeitsurteilen zuschlagen. Aber man darf sie auch nicht von der Wirklichkeit abschneiden, wie sie sich in Raum und Zeit entfaltet, und sie an einem anderen Ort als dem der phänomenalen Wirklichkeit verankern, jener aus subjektiven Handlungen bestehenden Wirklichkeit, aus denen das Gewebe des sozialen Lebens gemacht ist.

Das Ideal ist nicht einfach nur ein $»$ Mögliches $« .^{53}$ Es ist gewollt und nicht nur vorgestellt. Dieses Wollen ist nun auch hier wieder ein Indiz für eine aktive Bindung. Das Ideal konzentriert in sich eine Anziehungskraft, und diese Kraft bildet aus sich heraus eine bestimmte phänomenale Wirklichkeit, nämlich die der auf die Handlung übertragenen Spannung. Es ist gewollt, weil es von Subjekten getragen wird, die es wollen. In diesem Sinne ist es durchaus eine »lebendige Wirklichkeit $« .^{54}$ Es hat ein Eigenleben - was dazu verleiten kann, ihm eine losgelöste Existenz zuzuschreiben -, doch dieses Eigenleben unterscheidet sich nicht wirklich vom Leben der Subjekte, die sich daran binden und es in der Bewegung tragen, welche sie durch dessen Anziehungskraft einfängt. 
Man kann diese Naturalisierung des Ideals nicht genügend herausstellen. Denn von ihr hängt die spezifisch soziologische Bedeutung des Begriffs $\mathrm{ab}$, desgleichen die Anwendung einer Methode, die sie der Erkenntnis zugänglich macht. Genauer gesagt, ist hier eine Physik von Kräften impliziert, die zum Kriterium die Stärke der Bindung erhebt. Die Frage nach den Werten ist in einem tieferen Sinne die nach den sozialen Wertungsprozessen, gemessen und verglichen im Blick auf die Variation ihrer Stärke. Und diese Variation ist nicht mehr nur die der inhaltlichen Variation der Werte selbst, sondern die der ihr zugrunde liegenden affektiven und volitiven Bewegungen, unter Berücksichtigung des bereits hervorgehobenen Bindungsaspekts. Kurz, eine bestimmte Sichtweise der sozialen Physik gelangt hier wieder zu ihrem Recht, aber auf einem anderen Gebiet als der Morphologie - nämlich unmittelbar auf dem Gebiet der Vorstellungen, da sie nun mit einer messbaren und analysierbaren normativen Dimension ausgestattet sind. Wenn es daher zutrifft, dass die Soziologie im Ideal »ihr eigenes Reich ${ }^{55}$ erkennen muss, so muss man denn auch sagen, dass die Positivität der sozialen Phänomene ihr nicht die Aufzeichnung einer objektiven Gegebenheit zuweist. Vielmehr überantwortet sie ihr die Beschreibung sozialer Dinge im Sinne von Anreicherungsprozessen, in denen die Natur "gewissermaßen über sich selbst hinauswächst«..$^{56}$

Das ist der Kern der Durkheimschen Argumentation. Die Werte liegen in den sozialen Dingen, diesen natürlichen Dingen, in denen die Natur über sich selbst hinausgeht. Diese Überschreitung führt uns, weil sie Selbstüberschreitung ist, nicht aus der Natur heraus, wenn wir sie zu erforschen versuchen. Daraus ergibt sich die Position bezüglich der Werturteile: Einerseits lassen sie sich nicht auf die Wirklichkeitsurteile zurückführen; andererseits darf man sie nicht radikal davon trennen. Obwohl verschieden, basieren sie doch auf demselben Urteilsvermögen, das in beiden Fällen auf natürliche Realitäten angewendet wird. Nur durch die Art und Weise, wie das Gegebene gegeben ist, unterscheidet sich das Ideal von der schlichten Tatsache. Im Fall des Ideals ist die Überschreitung oder Umwandlung der eigentliche Gegenstand des Urteils, das sich nicht mehr auf die Feststellung dessen, was ist, beschränkt, sondern eine Realität ganz neuer Art beurteilt - eine »angereicherte«, mit einem Wert versehene Realität, die nichts anderes ist als der Prozess, durch den sie in ihrer Natürlichkeit über sich hinausgeht.

Ohne Abstriche an seinem Realismus, ja ihn sogar noch vertiefend - indem er sich auf jene sozialen Dinge konzentrierte, die sich manchen unserer Urteile als Werte aufdrängen -, eröffnet Durkheim in der Soziologie neue Perspektiven, die der Objektivismus seiner früheren Arbeiten, von den Regeln bis hin zu Über soziale Arbeitsteilung, nicht erahnen ließ. Und zwar indem er den Begriff der Gesellschaft auf eine bestimmte Bedeutung des "sozialen Lebens" bezieht, an dem das Leben der Individuen wirklich beteiligt ist und das zwar nicht auf das Leben seiner Bestandteile zurückgeführt werden kann, sich aber erst durch deren Leben aktualisiert, durch deren Fähigkeit, dieses Leben beständig zu speisen, in einer Bewegung nach Art einer Innervation, von der die Werturteile und die dadurch gebotenen moralischen Handlungen zeugen. Durkheims Soziologie der Moral ist eine Soziologie der moralischen Tatsachen, die in einer inneren Verbindung zu Urteilen und Handlungen stehen und an denen die sozialen Subjekte daher aktiv beteiligt sind. Wenn sie "externalistisch" erscheint, so weil sie deren subjektiven Status neu definiert, indem sie eine Spaltung in das Subjekt einführt, die sich nicht durch eine Verinnerlichung 
äußerer Normen auflöst, sondern eine gewisse Externalität gegenüber sich selbst zur Triebfeder der moralischen Spannung macht, bestimmt durch die stets aktuelle Koexistenz des Guten und der Pflicht in ihrer strukturierenden Widersprüchlichkeit. In Gesellschaft leben heißt gewiss, sich einer äußeren Ordnung zu unterwerfen. Doch angesichts des äußerlichen Charakters dieser Ordnung konzentriert sich das ganze Problem dort und stellt sich zunächst im Subjekt als eine seiner Dimensionen dar.

Dieser Widerstand gegen einen reinen Objektivismus findet seinen Ausdruck letztlich in der Ablehnung der utilitaristischen Auffassung, für die das Leben des Gesellschaftskörpers den höchsten Wert darstellt. Wenn man sich die Gesellschaft vorstellt "als ein System von Organen und Funktionen, das sich gegenüber den zerstörerischen Ursachen, die von außen auf sie einstürmen, zu behaupten sucht « ${ }^{57}$, so scheint das Leben der Gruppe nicht mehr zu sein als eine Reihe geeigneter Reaktionen auf Umgebungsreize. In dieser Auffassung wird jedoch ein Element vollständig ausgeblendet, ohne das schon der Begriff des Lebens in seiner Anwendung auf die Gesellschaft jeden Sinn verliert, nämlich das des »inneren moralischen Lebens«, wie Durkheim dies nennt. ${ }^{58}$ Trotz seiner spiritualistischen Konnotationen darf der Ausdruck nicht täuschen. Da dieses Leben aus Idealen und Werten besteht, ist es Gegenstand einer Wissenschaft, denn Ideale und Werte sind natürliche Realitäten, die als Dinge erfasst werden können. Die Mehrdeutigkeit beruht letztlich auf der Art, wie dieser Ausdruck dekliniert wird. Es gibt nur dann ein moralisches Leben, es gibt nur dann moralische Urteile und Handlungen, die von den sozialen Subjekten getroffen und vollzogen werden, wenn die Werte einen greifbaren Charakter annehmen, wenn also greifbare Phänomene sie umsetzen und objektivieren. Durch die Betonung dieses Aspekts der moralischen Realität tritt ihr Realitätscharakter deutlicher hervor und der externalistische Determinismus scheint die Oberhand zu gewinnen. Doch der Begriff des sozialen Dings erfordert mehr. Sobald die Objektivität der Werte der permanenten Schöpfung der Ideale untergeordnet wird, deren Herd das innere moralische Leben ist, wird es zu einem Wesensmerkmal der sozialen Dinge, dass sie sich bewegen, weil ihr Wert in einem Zusatz besteht, einer realen Anreicherung und damit in einer Verwandlung des sinnlich Gegebenen - einer Verwandlung, die Durkheim mit Hilfe mehrerer Kategorien zu umschreiben versucht, als deren Epizentrum das Heilige gelten kann.

47 Aus der Sicht der Moral, die voraussetzt, dass man auf die subjektiven Beiträge zurückgreift, die eine unverzichtbare Dimension darstellen, ist es diese Verwandlung, die man am Werk sieht, sobald sie sich vollzieht. Wenn das innere moralische Leben sich nicht auf ein rein physisches Geschehen reduzieren lässt, so weil es die Gesellschaft auf einer bestimmten Ebene als einen in Bewegung befindlichen Körper sichtbar macht, in Bewegung gesetzt durch Ideale, die überdies konstitutiv hinsichtlich einer von ihnen verwandelten aktuellen Realität sind. Anders als man meinen könnte, vermag man aus der Perspektive einer Soziologie der Moral - gerade weil sie sie sich nicht zum Objektivismus entschließen kann - am deutlichsten zu erkennen, wie das Soziale entsteht oder wie die sozialen Dinge hervorgebracht werden. Gewiss nicht als Früchte einer freien und an keine Regeln gebundenen Schöpfung, sondern als Modifikation der Wirklichkeit auf jener Ebene, auf der sie sich als Ensemble aus Handlungen und Urteilen über Handlungen entfaltet. Oder auch: Durch Handeln im Sinne von sozialem Handeln geht die Natur über sich selbst hinaus. 


\section{BIBLIOGRAPHIE}

Boltanski, L. / Thévenot, L. (2007 [1991]): Über die Rechtfertigung. Eine Soziologie der kritischen Urteilskraft, übers. von A. Pfeuffer, Hamburg: Hamburger Edition.

Durkheim, E. (1967): Soziologie und Philosophie, übers. von E. Moldenhauer, Frankfurt/M.: Suhrkamp.

Durkheim, E. (1967 [1906]): »Bestimmung der moralischen Tatsache«, in: Ders. (1967), S. 84-136.

Durkheim, E. (1967 [1911]): »Werturteile und Wirklichkeitsurteile«, in: Ders. (1967), S. 137-157.

Durkheim, E. (1970 [1898]): »L'individualisme et les intellectuels«, in: Ders.: La science sociale et l'action, hg. von J.-C. Filloux, Paris: PUF, S. 261-278.

Durkheim, E. (1975): Textes, 3 Bde., hg. von V. Karady, Paris: Ed. de Minuit.

Durkheim, E. (1975 [1887]): »La science positive de la morale en Allemagne«, in: Ders. (1975), Bd. 1, S. 267-343.

Durkheim, E. (1981 [1912]): Die elementaren Formen des religiösen Lebens, übers. von L. Schmidts, Frankfurt/M.: Suhrkamp.

Durkheim, E. (1988 [1930]): Über soziale Arbeitssteilung. Studie über die Organisation höherer Gesellschaften, übers. von L. Schmidts, durchges. von M. Schmid, Frankfurt/M.: Suhrkamp.

Gurvitch, G. (1937): Morale théorique et science des mœurs, Paris: Alcan.

Isambert, F.-A. (1992): »Les avatars du >fait moral««, in: Ders.: De la religion à l'étique, Paris: Cerf.

Kant, I. (1956): Kritik der praktischen Urteilskraft, in: Ders.: Werke in sechs Bänden, Bd. IV, hg. von W. Weischedel, Wiesbaden,.

Karsenti, B. (2006): La société en personnes. Études durkheimiennes, Paris: Economica.

Pharo, P. (2004): Morale et sociologie, Paris: Gallimard.

Rauh, F. (1890): Essai sur le fondement métaphysique de la morale, Paris: Alcan.

Rauh, F. (1903): L'expérience morale, Paris: Alcan.

\section{NOTES}

1. Durkheim (1975 [1887]).

2. Isambert (1992), S. 358.

3. Isambert (1992), S. 392.

4. Pharo (2004), S. 134.

5. Isambert (1992), S. 368; Pharo (2004), S. 98. Als erster verwies auf dieses Dilemma P. Gurvitch (1937), auf der Grundlage einer vergleichenden Lektüre von Durkheim und Rauh (1903). Gurvitch gelangt allerdings, wie Isambert gezeigt hat, eher zu einer Trennung der beiden Aspekte des Dilemmas als zu dessen Auflösung.

6. Durkheim (1967 [1906]) und Durkheim (1967 [1911]).

7. Siehe Durkheim (1975), Bd. 2, S. 257-288.

8. Durkheim (1975), S. 271. 
9. Zur Unterscheidung zwischen repressiver und stärkender Sanktion siehe Karsenti (2006), Kap. V und VI.

10. Durkheim (1967 [1906]), S. 93.

11. Pharo (2004), S. 99.

12. Durkheim (1988 [1893]), S. 124.

13. Durkheim (1967 [1906]), S. 95.

14. Durkheim (1967 [1906]), S. 85.

15. Durkheim (1967 [1906]), S. 85.

16. Kant (1956 [1788]), S. 196.

17. [leichte Veränderung der Übersetzung; A.d.Ü.].

18. Kant (1956 [1788]), S. 200.

19. Rauh (1890), S. 4. Diesem Buch folgte 1903 L'expérience morale, in dem Durkheims Thesen genauer diskutiert werden.

20. Durkheim (1967 [1906]), S. 85.

21. Durkheim (1967 [1906]), S. 96.

22. Durkheim (1967 [1906]), S. 105 f.

23. Durkheim (1967 [1906]), S. 97.

24. Kant (1954 [1788]), S. 200.

25. Kant (1954 [1788]), S. 202.

26. Durkheim (1967 [1906]), S. 98.

27. Durkheim (1975), Bd. 1, S. 404.

28. Zum Heiligen siehe meine Analyse in Karsenti (2006), Kap. IX.

29. Siehe Durkheim (1970 [1898]).

30. Durkheim (1967 [1906]), S. 100 [in der dortigen Übersetzung: »Neigung«, A.d.Ü.].

31. Durkheim (1967 [1906]), S. 105.

32. Durkheim (1967 [1906]), S. 106.

33. Durkheim (1967 [1906]), S. 108.

34. Durkheim (1967 [1906]), S. 108.

35. F.-A. Isambert (1992) beschreibt dieses Paradoxon sehr gut, S. $364 \mathrm{f}$.

36. $\mathrm{Zu}$ diesem Punkt, der Durkheim zu einer strengeren begrifflichen Unterscheidung zwischen Individuum und Person veranlasst, siehe Durkheim (1981 [1912]), S. 367 f.; sowie meinen Kommentar in Karsenti (2006), Kap. IX.

37. Durkheim (1967 [1906]), S. 118.

38. Durkheim (1967 [1906]), S. 120.

39. Durkheim (1969 [1911]), S. 137-157. Zu Durkheims Position, in den zeitgenössischen Kontext einer allgemeinen Erörterung über Werte eingebracht, siehe Gurvitch (1937), S. 68 ff.

40. Durkheim (1969 [1911]), S. 137.

41. Durkheim (1969 [1911]), S. 138.

42. Durkheim (1969 [1911]), S. 138.

43. Man denke hier vor allem an das Buch von Boltanski / Thévenot (2007 [1990]).

44. Durkheim (1969 [1911]), S. 139.

45. Ein strategisches Verb, das 1914 im Zentrum der Durkheimschen Kritik am Pragmatismus stehen wird.

46. In einer wichtigen Fußnote in Durkheim (1981 [1887]), 287 f. (Fn. 6), macht Durkheim das Problem der Autorität zum soziologischen Problem par excellence, fundamentaler noch als das des Zwangs, dem er in den Regeln der soziologischen Methode noch den Vorrang eingeräumt hatte.

47. Durkheim (1969 [1911]), S. 141.

48. Durkheim (1969 [1911]), S. 140.

49. Ab 1911 konzentriert Durkheims Argumentation sich auf das Phänomen der Verausgabung, der zweckfreien und kostspieligen Aktivitäten, um darauf eine gegen den Utilitarismus 
gerichtete Argumentation zu entwickeln, die manche später von Mauss in Die Gabe vorgebrachten Thesen vorwegnehmen. Vor allem frappiert die Formulierung: „Selbst das wirtschaftliche Leben hält sich nicht streng an die Regeln der Ökonomie« (Durkheim [1969 (1911)], S. 144).

50. Durkheim (1969 [1911]), S. 144.

51. Durkheim (1969 [1911]), S. 149 f.

52. Durkheim (1969 [1911]), S. 147.

53. Durkheim (1969 [1911]), S. 148.

54. Durkheim (1969 [1911]), S. 148.

55. Durkheim (1969 [1911]), S. 156.

56. Durkheim (1969 [1911]), S. 157.

57. Durkheim (1969 [1911]), S. 149.

58. Durkheim (1969 [1911]), S. 150 [in der dt. Übers.: »innere Sittlichkeit«, A.d.Ü.].

INDEX

Mots-clés : sociologie morale, Emile Durkheim

Schlüsselwörter : Soziologie der Moral, Emile Durkheim

\section{AUTEURS}

\section{BRUNO KARSENTI}

Bruno Karsenti (geb. 1966), Philosoph und Soziologe. Nähere Informationen finden Sie hier. 